\title{
CÓMO OPTIMIZAR LA GESTIÓN PÚBLICA DEL PERÚ
}

Luis Fernando Valeriano Ortíz*

Ifernandovaleriano@yahoo.es

\section{RESUMEN}

El Sistema Educativo Nacional constituye el cimiento fundamental para el desarrollo socio-económico del país, en consecuencia requiere de un proceso de reingeniería para su modernización de acuerdo con el avance de la ciencia, tecnología y humanidades, dentro de un contexto de mundialización.

Palabra clave: Sistema Educativo Nacional.

\section{ABSTRACT}

The national education system is the fundamental foundation for socio-economic development therefore requires a reengineering process for modernization in accordance with the advancement of science, technology and humanities, within the context of globalization.

Keyword: National Education System.

\footnotetext{
* Lic. en Administración, Bach. en Derecho y Ciencias Políticas, Bach. en Sociología, Maestría en Educación con Mención Administración Universitaria y Doctor en Administración. Director de Escuela Académica Profesional en Administración períodos 1992 y 2002 FCA-UNMSM, Decano FCA-UNMSM Junio 2007-Mayo2010 Profesor Principal. Ex Gerente de Adminitración RTP S.A, ex Director Nacional de Personal del INAP.
} 


\section{EVOLUCIÓN DEL PENSAMIENTO ADMINISTRATIVO}

La ciencia administrativa a partir del siglo XX ha evolucionado en su marco teórico y práctico, lo que ha originado el surgimiento de nuevas herramientas en el proceso administrativo para optimizar la gestión empresarial, como es el caso del planeamiento estratégico, táctico y operacional aplicando las matrices para fines del diagnóstico empresarial y el Balanced Scorecard.

En materia de la organización surgen los modelos organizacionales, el comportamiento organizacional, el desarrollo organizacional y la cultura organizacional; la dirección se fortalece con el sistema de información gerencial para la toma de decisiones - proceso técnico relacionado con el desarrollo de las tecnologías de la información y comunicaciones-y la integración comprende al intangible más importante de una entidad, que es el talento humano y los procesos técnicos de competencias laborales, el reclutamiento y la selección de personal, así como el coaching, el mentoring y el consulting como herramientas modernas de la capacitación del personal, los procesos técnicos de evaluación de puestos, las escalas salariales y la evaluación del rendimiento del personal por productividad, los sistemas biométricos de control de asistencia del personal, que alimenta a la planilla de remuneraciones.

En materia de control éste se desagrega en control previo, concurrente y posterior, este último a través de las auditorías contables-financieras, administrativas y de gestión, así como los exámenes especiales, todos ellos orientados a mejorar la competitividad empresarial mediante el desarrollo de productos y prestación de servicios de calidad, tal como lo plantea Michael Porter (2010) para satisfacer las necesidad de los consumidores, usuarios o clientes según el giro empresarial. Este planteamiento técnico no es ajeno a la administración pública; por el contrario, debe ser tomado en cuenta para prestar un servicio de calidad, que es su razón de ser.

\section{ADMINISTRACIÓN ESTRATÉGICA}

En estas últimas décadas, la gestión empresarial se desarrolla sobre la base de la administración estratégica, siendo estos los autores de textos más representativos y estudiados en el sistema universitario: Fred David, Jones Hill, A. Thompson y G. Johnson.
Esta especialidad nace como consecuencia de que los países, al insertarse en la globalización de los mercados internacionales, toman en cuenta los factores del entorno (macro y micro) e intorno (áreas estratégicas) empresarial.

También la gestión pública tiene que tomar en cuenta estos procesos de modernización, principalmente aquellos sectores productivos involucrados en los tratados de libre comercio suscrito por el Perú con diversos países del mundo. Es en este contexto que las entidades de la administración pública deben tomar en cuenta este proceso técnico para optimizar su gestión:

- El entorno empresarial/institucional. Que se desagrega en macro entorno nacional e internacional con sus variables políticas, económicas (macro y micro), sociales, educativas, culturales, legales, etc., teniendo como fuentes de información a los informes sobre el Perú que han sido desarrollados por el Banco Mundial (BM), Banco Interamericano de Desarrollo (BID), Fondo Monetario Internacional (FMI), Banco Central de Reserva del Perú (BCRP) y el Instituto Nacional de Estadística e Informática (INEI), con una mirada retrospectiva de los últimos diez años, para analizar su comportamiento en términos de evolución o deterioro. El micro entorno empresarial/institucional se analiza tomando en cuenta las variables: clientes/ usuarios, competencia y los proveedores.

- El entorno empresarial/institucional. Constituido por las áreas estratégicas de la gestión empresarial/pública como son: la contabilidad y las finanzas, marketing y las ventas, producción u operaciones, y la gestión administrativa, que comprende al talento humano, la logística, los costos y presupuesto, etc.

En consecuencia, el desarrollo de la administración estratégica en el ámbito empresarial o de la gestión pública, toma en cuenta los factores, variables y los indicadores del entorno e intorno de la gestión, para establecer la visión, misión y los valores empresariales/ institucionales en un aspecto axiológico, para luego desarrollar el diagnóstico que está referido a la caracterización de la problemática empresarial, de la cual se desprenden los objetivos y sus actividades, las metas, las políticas y las estrategias, procesos que posibilitan su calendarización en el tiempo con su correspondiente evaluación y seguimiento de la gestión. 


\section{MODELOS ORGANIZACIONALES}

Es importante resaltar que la administración y el planeamiento estratégico constituyen la base fundamental para desarrollar los modelos organizacionales y, de esta forma, optimizar la gestión empresarial privada o pública, las cuales también han sido materia de evolución a lo largo del tiempo, como es el caso de los modelos organizacionales: funcionales, por clientes, por productos, por zonas geográficas, por procesos o redes, sistémicas, matriciales, virtuales y las llamadas organizaciones globalizadas como las multinacionales, multidivisionales, holdings, los clusters, etc.

Las entidades, en función a sus proyecciones de crecimiento, demandan modelos organizacionales flexibles y horizontales, que respondan a sus necesidades; en consecuencia, no podrían plantearse recetas organizacionales. Por el contrario: la organización de cada empresa responde a su perfil empresarial.

De igual forma, es relevante tomar en cuenta el comportamiento organizacional, el desarrollo organizacional y la cultura organizacional para la implementación de los modelos organizacionales, que contribuyan a mejorar la calidad de los productos y /o servicios que presta la entidad dentro del contexto de la competitividad empresarial.

\section{VENTAJA COMPETITIVA}

El Dr. Michael Porter en su obra "La Ventaja Competitiva - Creación y sostenibilidad de un rendimiento superior" (2010), centra su atención en las estrategias competitivas, cadena de valor, ventaja en costos, diferenciación de los productos, etc., orientados al valor que una empresa logra crear para sus clientes, mediante la calidad de sus productos y/o servicios que oferta, procesos técnicos que deben ser tomados en cuenta en el diseño de los modelos organizacionales para fortalecer la gestión empresarial y garantizar los márgenes razonables de utilidad y/o mejorar la prestación de los servicios que oferta.

\section{ADMINISTRACIÓN PÚBLICA}

Es impensable, tomando en cuenta la evolución del pensamiento administrativo a partir de F. Taylor y H. Fayol (1903) hasta la fecha, lo expuesto en el presente artículo y la globalización de los mercados, que la gestión pública de nuestro país persista en tener organizaciones verticales que son inflexibles y atomizadas, que frenan o desaceleran el crecimiento de la economía del país. Por el contario, el Perú debería proyectar planes de desarrollo a largo, mediano y corto plazo aprobados y concertados, debidamente financiados, donde los sectores productivos y de servicios estén contemplados con sus factores, variables e indicadores de crecimiento sostenible, para que los gobiernos de turno asuman compromisos con la sociedad peruana. No bastan las hojas de ruta, que son lineamientos de política sectorial; se requiere de una planificación integral y concertada. Esta propuesta posibilitaría que el sector empresarial del país y del extranjero, desagregadas sus actividades en empresas industriales, de servicios y comerciales, ejecuten sus programas de inversiones en aquellos sectores estratégicos establecidos en la planificación nacional para garantizar el crecimiento del país en forma sostenida en el tiempo y no sólo en función a recuperar su inversión.

La administración pública de nuestro país requiere de un proceso de reingeniería para modernizar su gestión, acción que puede demandar tiempo, pero que es necesaria ya que no se puede continuar con reformas parcializadas, desinteligentes y sin asignación de recursos presupuestales, que solo llevan a la indiferencia del trabajador público y al malestar general por parte de los usuarios de los servicios que presta. Es por ello que considero que es el momento de elaborar y poner en ejecución un Programa de Modernización de la Gestión Pública, en el que el capital humano -que es el intangible más importante de la gestión- asuma un rol de liderazgo, de trabajo en equipo, motivación y, fundamentalmente, de identidad, constituyéndose en la columna vertebral de este proceso, que posibilite el éxito de la gestión pública del país.

\section{LINEAMIENTOS PARA UN PROGRAMA DE REINGENIERÍA DE LA GESTIÓN PÚBLICA DEL PERÚ:}

1. Elaboración del plan estratégico y táctico para el sector público nacional y que sea participativo. Que comprende a los gobiernos central, regional y local en forma articulada, desagregado por sectores productivos y sociales con sus correspondientes proyectos de inversión y proyectos sociales, precisando su temporalidad, fijando sus objetivos, metas y estrategias, utilizando el Balanced Scorcard para su implementación. También es necesario 
implementar el Instituto Nacional de Planificación con una concepción moderna, dotada de personal altamente calificado y con los recursos necesarios. Es imprescindible que exista la voluntad política del Ejecutivo para concertar con los gobiernos regionales, en el sentido de recoger sus necesidades y las prioridades de los proyectos de inversión de los sectores productivos que demande cada región, para ser incorporados en la planificación nacional y asignarles un presupuesto. De igual forma, los gobiernos regionales y los gobiernos locales provinciales y distritales de la jurisdicción deberían concertar sus objetivos y metas para garantizar un desarrollo sostenido e integral en el ámbito de su competencia.

Es necesario promover la inversión nacional y extranjera dentro de una economía de mercado, respetando la legislación nacional y, fundamentalmente, la conservación del medio ambiente y la ecología, mediante la presentación de proyectos como requisito indispensable para las exploraciones y explotaciones de nuestros recursos no renovables y asumiendo compromisos de implementar programas de responsabilidad social con las comunidades nativas.

2. Desarrollar y poner en ejecución el proyecto de regionalización. Que no sólo se limite a la demarcación geográfica del país, que recoja la caracterización de la problemática presentada en cada uno de los gobiernos desde 1985 a la fecha, que han politizado y polarizado al país. Este proyecto debe orientarse a la creación de las macro regiones, tomando en cuenta la afinidad cultural de los pueblos, su interconexión con vías de comunicación terrestre y fluvial según sea el caso, y las potencialidades de recursos naturales de la Costa, Sierra Y Selva. Esta regionalización debe responder a una verdadera descentralización y desconcentración funcional y territorial sectorial del país, respetando los recursos económicos generados por cada región, sobre la base de un país que requiere un crecimiento integral y no centralista. Cada región debería formular su organización, teniendo como referencia su planificación regional articulada con la nacional, dando prioridad a los sectores sociales como la salud y educación regional, ejes del desarrollo nacional. Es importante, para fines estratégicos, generar polos de desarrollo para las regiones fronterizas, estudiando -en cada caso- la factibilidad de crear zonas francas comerciales e industriales, tomando en cuenta las experiencias de Brasil con Manaos y la realidad de Iquitos en el Perú.

3. Elaboración del presupuesto para el sector público nacional participativo. Sobre la base de la planificación nacional, que comprenda al gobierno central y a los gobiernos regionales con sus correspondientes sectores sociales y productivos, incrementando los ingresos (aumento de la presión tributaria, ya que todavía existen sectores informales que no cumplen sus obligaciones tributarias y sectores formales que las evaden, además de considerar los recursos económicos provenientes del canon y sus diversas fuentes como el minero, gasífero, etc.), y racionalizando los egresos (redistribuyendo el gasto sectorial en forma prioritaria de acuerdo a lo establecido en la planificación, asignando prioritariamente el 8\% en forma progresiva a la educación nacional y el $2 \%$ a los programas de ciencia y tecnología para fortalecer las investigaciones básicas y aplicadas que demanda el país, además deberá racionalizarse la organización del estado, identificando los organismos burocráticos e ineficientes comprendidos en ministerios, empresas del Estado, instituciones públicas descentralizadas, organismos reguladores, etc. Se requiere también optimizar los programas de inclusión social a nivel nacional y sus fuentes de financiamiento).

4. Capacitación y desarrollo para el trabajor de la administración pública. Implementando la Universidad Nacional de Gestión Pública para los grupos ocupacionales de directivos y profesionales, y la Escuela Superior de Administración Pública para los grupos ocupacionales de nivel técnico, administrativo y de servicios. El actual nivel educativo de los trabajadores de la administración pública demanda capacitación, especialización y perfeccionamiento, la que debe ser integral para todos los trabajadores, precisando las carreras profesionales y las líneas de capacitación a atenderse en función a la planificación nacional y regional. También es necesario promover una nueva legislación laboral para los trabajadores de la administración pública, que contribuya a elevar los indicadores de eficiencia y eficacia laboral en la prestación de los servicios, además de modernizar la actual legislación laboral pública que tiene muchas décadas de vigencia.

5. Diseño de modelos organizacionales horizontales. Que suplan a las organizaciones verticales vigentes, llegando solo hasta el tercer nivel 
de la desagregación organizacional, simplificando procedimientos sustantivos y adjetivos mediante los sistemas de información gerencial, unificando los programas informáticos para agilizar la toma de decisiones.

Es importante racionalizar la organización del Estado, dando prioridad a los sectores de educación, salud, y a los sectores productivos estratégicos. También se deben racionalizar las escalas remunerativas de los trabajadores de la administración pública por grupos ocupacionales y puestos de trabajo simplificados, sobre la base de una remuneración básica real y no minimizada a montos irrisorios como en la actualidad.

6. Implementar el sistema de información gerencial. Utilizando equipamiento y programas informáticos modernos y estandarizados para la gestión pública, proceso técnico que garantizaría contar con información real y virtual para la toma de decisiones por parte de las autoridades del gobierno, fundamentalmente aplicado a los sistemas administrativos de planificación, presupuesto, tesorería, abastecimiento, tributación, estadístico, jurídico, cooperación internacional, comercio exterior; a los sectores productivos y de servicios, y a los gobiernos regionales y locales. La implementación de las tecnologías de información y comunicaciones en el sector público nacional fortalecería el desarrollo nacional.

7. Desarrollo de los programas de control previo y posterior. Mediante el fortalecimiento del Sistema Nacional de Control y su organismo rector, que es la Contraloría General de la República. La función de control debe ser repotenciada, descentralizada y debe mantener la independencia de los poderes del Estado para guardar el equilibrio y transparencia de la gestión pública; para tal efecto se requiere de profesionales multidisciplinarios especializados en la acción de control, que no tengan antecedentes judiciales y administrativos concluidos o en proceso, y menos que tengan incompatibilidades laborales. La función de control posterior no sólo debe circunscribirse a evaluar la gestión contable-financiera por mandato legal, sino también a los sistemas administrativos y a la razón de ser de una institución, por tanto es integral en el ámbito institucional. El profesional que labora en el sistema nacional de control debe tener muy claro que el intangible más importante en las organizaciones es el capital humano y estamos en la era de la gestión del conocimiento; en consecuencia, es el punto de partida para toda acción de control.

8. Fortalecer el sistema democrático del país. A través de dar el marco regulatorio para la no reelección de autoridades, que incluya al presidente de la República, a los congresistas, presidentes regionales, alcaldes y de toda autoridad elegida, de esta forma se consolida el sistema democrático del país y se moderniza a los partidos o agrupaciones políticas, posibilitando que sus cuadros de profesionales asuman nuevas responsabilidades. La alternancia en el poder minimiza la corrupción, además nadie es imprescindible en la gestión pública.

También deben estar comprendidos en la norma los cargos designados políticamente en todo el aparato estatal. Es importante resaltar que los cargos de presidente del Banco Central de Reserva, presidente de la Superintendencia de Banca y Seguros, y el de contralor general de la República deberían estar ocupados por profesionales del más alto nivel académico, con experiencia laboral especializada acreditada y que no formen parte de ningún partido político.

Definitivamente debe modificarse la Constitución Política del país, en los artículos correspondientes a los requisitos mínimos para ser elegido congresista de la República en razón a su función legislativa y fiscalizadora que cumplen. Nada justifica la situación actual; por el contario, no se encuentra explicación de que existan tantas universidades públicas y privadas al interior del país formando profesionales para continuar en un estatus quo que afecta la gobernabilidad del país.

9. Modernizar la Ley Universitaria y la Ley General de Trabajo. Estos son dos dispositivos legales que se han quedado congelados en el tiempo por intereses políticos y que frenan el desarrollo del país. El Perú requiere de una legislación universitaria moderna, acorde con la evolución de la ciencia, tecnología y las humanidades en un mundo globalizado y competitivo, debidamente financiada sino es letra muerta, otorgando plazos a las actuales universidades públicas y privadas del país para que desarrollen sus procesos de autoevaluación, evaluación externa, acreditación y certificación, que respondan a estándares de calidad por cada carrera profesional. Se debe frenar el mercantilismo de la formación profesional en el país. 
Es relevante contar con una legislación laboral para la actividad pública y privada, que responda al desarrollo del país. El trabajador no es una pieza más dentro del engranaje de una maquinaria, no es un insumo o materia prima; por el contrario, es un ser pensante que requiere de un marco legal justo y equitativo que garantice sus deberes y derechos laborales.

Deben respetarse los convenios colectivos y el trabajador debe contar con un sistema pensionario que le permita una calidad de vida decorosa para su jubilación, de acuerdo a ley. Lamentablemente el trabajador peruano tiene que laborar hasta la culminación de su ciclo de vida, por las irrisoria pensiones con límites que otorga el Estado y por las altas comisiones que son transferidas al sistema privado de pensiones, deducidas de las remuneraciones del trabajador en su condición de personal activo, teniendo como límite pensionario sus aportes.

10.Comercio exterior. Nuestro país viene consolidando los factores e indicadores de la macro economía. Nuestra balanza comercial es positiva y refleja que tenemos más exportaciones que importaciones, lo cual es saludable para el desarrollo nacional. Existen varias explicaciones para esta situación positiva: la inserción del país en la globalización de los mercados internacionales, los tratados de libre comercio suscrito con países desarrollado y en vías de desarrollo, las exportaciones de productos tradicionales como los minerales y los no tradicionales como los productos de agroindustria, permitiendo el ingreso de divisas. Esta situación requiere ser repotenciada con estrategias comerciales en las que el sector privado apueste por la calidad y competitividad, contando para ello con la participación de nuestras embajadas en el exterior mediante los agregados comerciales, quienes deben ser el vínculo entre los gobiernos y empresas privadas extranjeras y el sector empresarial privado del país, teniendo una visión para extenderse a sectores estratégicos como son el turismo y la gastronomía.

11.Reingeniería del sistema educativo del país. Los fracasos sistemáticos de los diferentes gobiernos en materia educativa -reflejados en la aplicación del proyecto educativo nacional y en la frondosa legislación del sistema educativo, sin el financiamiento respectivo, y lo que es más grave, el desconocimiento de la problemática de la educación del país, abordando solo aspectos aislados del sistema educativo como la infraestructura educativa, duplicar las remuneraciones, dictar leyes de la carrera magisterial para incentivar la meritocracia individual del docente, etc.-, contribuyen a los niveles en que se encuentra la educación del país en la actualidad. La probable solución es abordar el problema en forma integral: desarrollando el proyecto educativo nacional que recoja el éxito de los modelos educativos de los países asiáticos y su articulación con la legislación magisterial, que considere el incremento del presupuesto de la educación a un $8 \%$ proyectado a 10 años; además de la revisión y modernización de los planes de estudios de los niveles inicial, primario, secundario, de instituciones superiores no universitarias y del sistema universitario, bajo el contexto de una estructura curricular flexible orientada al emprendimiento, que responda a la realidad de cada región y que recoja los avances de la ciencia, tecnología y las humanidades. Cuando se logren concretar estas líneas de acción recién se estaría en la capacidad de desarrollar un programa de capacitación intensivo para los docentes en función al nuevo plan curricular con una proyección de cinco años; diseñar infraestructuras estandarizadas para la Costa, Sierra y Selva tomando en cuenta la población estudiantil; dotar de laboratorios y talleres con tecnología de punta; promover la producción intelectual del docente y el desarrollo de trabajos de investigación. En este contexto no hay espacio para la corrupción y mediocridad.

12.Descentralización del Poder Judicial. Para que la administración de justicia sea eficiente es necesario dotarla de los recursos necesarios, fundamentalmente de una capacitación a nivel de postgrado para los magistrados y fiscales en especialidades como derecho penal, civil, laboral, tributario, comercial, administrativo y del lavado de dinero, sin olvidar los aspectos de gestión administrativa y económica judicial.

La descentralización del Poder Judicial necesariamente debería implementarse ya que la carga procesal actual se refleja en el hacinamiento de los centros penitenciarios. Deberían crearse más salas de la Corte Suprema con sedes en macro regiones; las Cortes Superiores y los juzgados deberían implementarse en función a la densidad demográfica de las provincias y distritos. Los magistrados deberían ser evaluados permanentemente con fines de su ratifación en el cargo, para garantizar su probidad y capacidad profesional, además de sancionar 
a los magistrados que acrediten inconductas funcionales, en plazos perentorios.

El trabajador que labora en el Poder Judicial debe ser evaluado para verificar sus competencias laborales en el puesto de trabajo.

13* Moralización de la gestión pública. Debería ser una política de Estado sobre la base de decir no a la impunidad y a la prescripción de los delitos o faltas cometidas por los funcionarios y trabajadores de la administración pública, respetando el debido proceso y el derecho a la defensa. Corresponde a la Contraloría General de la República proponer las sanciones disciplinarias en la vía administrativa, y al organismo del Estado le corresponde aplicar las sanciones disciplinarias, creando el Tribunal del Servicio Civil para acudir en vía de apelación en última instancia administrativa.

De igual forma corresponde al Poder Judicial resolver en la vía penal, civil y laboral los casos que, por su naturaleza, le corresponda sancionar. Es posible que este planteamiento exista pero es letra muerta: el país requiere de una sociedad con valores y con identidad nacional, y no al borrón y cuenta nueva.

Las opiniones vertidas y los planteamientos técnicos expuestos podrían servir para dar origen a diversos proyectos para cada tema y sector. Debido a su importancia y complejidad no pretendo haber analizado a todas las áreas de la gestión pública y menos ser la solución para los problemas existentes, es simplemente una modesta opinión profesional que a mi juicio puede ser tomada en cuenta por quienes tienen la responsabilidad de la conducción del país, de estimarse pertinente.

\section{CONCLUSIONES}

El Perú viene consolidando su crecimiento macro económico en esta última década, el sistema democrático se fortalece con la alternancia de autoridades, sus exportaciones tradicionales y no tradicionales repuntan en los mercados internacionales, el flujo de inversiones nacionales y extranjeras se incrementan, sin embargo todavía quedan sectores sociales en pobreza y extrema pobreza que requieren ser atendidos con los programas de inclusión social y la inseguridad ciudadana se acentúa.

Corresponde al gobierno modernizar la gestión pública para brindar un servicio eficiente y eficaz a la sociedad peruana y contribuir al desarrollo del país, donde el sistema educativo nacional sea la prioridad de la reactivación.

\section{LITERATURA CITADA}

Banco Mundial, Informe: Perú en el umbral de nueva era, lecciones y desafíos para consolidar el crecimiento económico y un desarrollo más incluyente. Volumen I (162 páginas), II (267 páginas), III (357 páginas), IV (486 páginas) y V (673 páginas).

Consejo Nacional de Educación. Proyecto Educativo Nacional al 2021, (145 páginas).

Ley del Servicio Civil N*30057. 